\title{
Electroconvulsive therapy in a twelve-year-old boy with catatonic symptoms
}

\author{
Reza Bidaki, Tahere Sadeghye, Masoud Amin, Omid Rezahosseini
}

\section{Summary}

Studies Electroconvulsive therapy (ECT) is effective in the treatment of several psychiatric illnesses. Only a few studies describe the use of ECT in children and adolescents. We describe the treatment of catatonic symptoms with ECT in a twelve-year-old boy who presented with complaints of nausea, vomiting and ataxia and developed catatonic symptoms 48 hours after admission to hospital. The response to diazepam infusion was partial but recovery was complete after 12 treatments of ECT. There were no adverse effects to ECT.

SL J Psychiatry 2013; 4 (1):20-21

\section{Introduction}

Oral Electroconvulsive therapy (ECT) is effective in the treatment of several psychiatric illnesses (1) and is commonly used in the treatment of severe or treatmentresistant mood disorders and catatonic syndromes (9). Development of guidelines on the administration of ECT has resulted in an improvement in safety and in the minimization of side effects. However, only a few studies describe the use of ECT in children and adolescents $(2,3)$. In this age group, ECT has mostly been used in the treatment of mood disorders.

Electro-convulsive therapy is an effective and relatively safe procedure in high-risk special patient populations such as children and adolescents, pregnant females, elderly and the medically ill (4). Disorders of the central nervous system and cardiovascular and respiratory systems should be excluded prior to the administration of ECT.

Catatonia is a rare but serious condition which is caused by mood disorders, schizophrenia, neurological and other organic conditions $(5,6)$. Characteristic features of catatonia include motor immobility, posturing, stereotyped movements, motor excitement, mutism, negativism, stupor, and reduced food or fluid intake which can compromise physical health. Catatonia is usually an acute condition, although chronic catatonia persisting for many months has been described (7).

Benzodiazepines are recommended as the first line treatment for catatonia because they are effective and safe. ECT is indicated when symptoms do not resolve with benzodiazepines (8). In addition, underlying medical conditions, if any, must be excluded or confirmed and treated. In this report, we describe the treatment of catatonic symptoms with ECT in a twelveyear-old boy.

\section{Case report}

A twelve-year-old boy was admitted to a pediatric neurology ward with complaints of nausea, vomiting and ataxia. Forty eight hours after admission, he developed mutism, rigidity and refused all food and drink. Shortly afterwards, he became stuporous. There was no past history or family history of psychiatric illness. Apart from the presence of catatonic symptoms, physical examination was normal.

The differential diagnoses of encephalitis, a space occupying lesion, metabolic abnormalities and druginduced catatonia were considered. The following investigations were done; serum copper $=139$ $\mu \mathrm{mol} / 1$, Serum ceruloplasmine $=35.5 \mathrm{mg} / 1$, Widal Test -Negative, Thyroid functions; T3=1.5 nmol/1, $\mathrm{T} 4=10.5 \mu \mathrm{g} / \mathrm{dl}, \mathrm{TSH}=1.1 \mathrm{mIU} / 1, \mathrm{SGOT}=37 \mathrm{IU} / 1$, $\mathrm{SGPT}=17 \mathrm{IU} / 1$, Anti helicobacter $(\mathrm{Ig} \mathrm{G})=6.3 \mathrm{IU} /$ $\mathrm{ml}$, Anti helicobacter $(\operatorname{Ig} \mathrm{A})=7.8 \mathrm{IU} / \mathrm{ml}$, Anti thyroglobulin $=1.1 \mathrm{IU} / \mathrm{ml}$, Anti-Tpo= $=0.1 \mathrm{IU} / \mathrm{ml}$ Ammonia $=0.4 \mu \mathrm{g} / \mathrm{dL}$

All biochemical investigations including a cerebrospinal fluid analysis and a urinary drug screen yielded negative or normal results. A MRI scan and the EEG were unremarkable.

As there was no evidence of an organic aetiology, the patient was treated with a diazepam infusion. Thereafter, he responded to stimulation with eye opening and verbal responses.

As recovery was inadequate, ECT was administered on consecutive days. There was no significant change in the clinical state after three treatments. Further treatment with ECT was continued every other day. Perphenazine was commenced after the fourth treatment. It was gradually increased to $20 \mathrm{mg} /$ day. After the fifth treatment an improvement in the clinical state was noted. After ten treatments, the patient was alert and oriented. He was eating well, motor symptoms of catatonia had disappeared and vomiting had stopped. Upper gastrointestinal endoscopy was normal. A total of 12 treatments were administered.

At the time of discharge the patient was well. There was no vomiting, impairment of memory, mood symptoms or psychotic symptoms. Perphenazine $20 \mathrm{mg}$ /day was continued. He continued to be well one month after discharge. Repeat investigations including EEG and MRI scan were normal. 


\section{Discussion}

Catatonic symptoms can occur due to organic causes. As this patient presented with nausea, vomiting and ataxia, an organic cause was considered and excluded. He did not have an adequate response to diazepam but recovered completely with ECT.

There is evidence that benzodiazepines and ECT are effective and safe in the treatment of catatonia (10). However there are concerns regarding the use of ECT in children and adolescents. Studies have shown that adverse effects of ECT in adolescents are the same as that in adults (11).

The American Academy of Child and Adolescent Psychiatry best practice treatment parameters for ECT in adolescence recommend memory evaluation at the onset and the end of acute treatment, and then again three to six months after ceasing ECT.

The use of succinylcholine in the pediatric population for neuromuscular blockade in ECT poses a theoretical risk of malignant hyperthermia and succinylcholine carries a Federal Drug Administration warning for pediatric usage in the United States (9).

A review of Pubmed records from 1985 to 2009 found 59 cases of children and adolescents with catatonia treated with ECT. Response to ECT was favorable in 45 patients, with partial improvement noted in three and lack of response recorded in only one patient (9).

There are reports from Asian countries on the use of ECT in the treatment of catatonia in children and adolescents. In a retrospective study in North India, ECT was found to be effective in the treatment of severe psychiatric disorders in adolescents and the frequency of adverse effects were similar to that in adults (11). In Iran , Esmaili et al. reported a case of a six-year-old girl suffering from major depressive disorder with catatonic features, treated with ECT (12).

Wider recognition of the benefits of ECT in children and adolescents with catatonia will lead to the consideration of ECT as a viable treatment option in this age group (13).

\section{Declaration of interest}

None declared

Reza Bidaki, Ass Professor of Psychiatry

Rafsanjan University of Medical Sciences, Rafsanjan, Iran

Tahere Sadeghye, Ass Professor of Child and

Adolescence Psychiatry, Isfahan University of Medical

Sciences, Iran

Masoud Amin, Medical student. Rafsanjan University of

Medical Sciences, Rafsanjan, Iran

Omid Rezahosseini, Rafsanjan University of Medical

Sciences, Rafsanjan, Iran

Corresponding author: Reza Bidaki

Email:Reza_Bidaki@yahoo.com

\section{References}

1. Nakatake $M$, Teraishi $T$, Ide et al. Modified electroconvulsive therapy for recurrent major depressive disorder in a meningioma patient: a case report of clinical experience. Hukuoka acta medica. 2010;101(9):198-206

2. Bertagnoli MW, Borchardt CM. A review of ECT for children and adolescents. J Am Acad Child Adolesc Psychiatry 1990; 29(2):302-7

3. Wachtel LE, Dhossche DM, Kellner CH. When is electroconvulsive therapy appropriate for children and adolescents? Med Hypotheses 2011;76(3):395-9

4. Rabheru K. The use of electroconvulsive therapy in special patient populations. Can J Psychiatry 2001;46(8):710-9

5. Cohen D, Flament M, Dubos PF, Basquin M. Case series: catatonic syndrome in young people. J Am Acad Child Adolesc Psychiatry 1999;38(8):1040-6

6. Taylor MA, Fink M. Catatonia in psychiatric classification: a home of its own. Am J Psychiatry 2003;160(7):1233-41

7. Ghaziuddin N, Gih D, Barbosa V, Maixner DF, Ghaziuddin M. Onset of catatonia at puberty: electroconvulsive therapy response in two autistic adolescents. J ECT 2010;26(4):274-7

8. Bush G, Fink M, Petrides G, Dowling F, Francis A. Catatonia. I. Rating scale and standardized examination. Acta Psychiatr Scand 1996;93(2):129-36

9. Consoli A, Benmiloud M, Wachtel L, Dhossche D, Cohen D, Bonnot O. Electroconvulsive therapy in adolescents with the catatonia syndrome: efficacy and ethics. J ECT 2010; 26(4):259-65

10. Shukla L, Narayanaswamy JC, Gopinath S, Math SB. Electroconvulsive therapy for the treatment of organic catatonia due to viral encephalitis. J ECT 1997; 28(3) e27-8

11. Grover S, Malhotra S, Varma S, Chakrabarti S, Avasthi A, Mattoo SK. Electroconvulsive Therapy in Adolescents: A Retrospective Study From North India. J Ect. 2013; 29(2)122-6

12. Esmaili T, Malek A. Electroconvulsive therapy (ECT) in a six-year-old girl suffering from major depressive disorder with catatonic features. Eur Child Adolesc Psychiatry 2007;16(1):58-60

13. Ghaziuddin N, Dhossche D, Marcotte K. Retrospective chart review of catatonia in child and adolescent psychiatric patients. Acta Psychiatr Scand 2011;125(1):33-8 